\section{Original Article}

Dr. Md. Zahid Hossain

BDS, PGD \& PhD (Japan)

Assoc. Professor \& Chairman

Division of Periodontics

Dept. of Preventive Dental Sciences

College of Dentistry, King Khalid University

Kingdom of Saudi Arabia

Dr. Hytham N. Fageeh

BDS

Intern Doctor

Division of Periodontics

Dept. of Preventive Dental Sciences

College of Dentistry, King Khalid University

Kingdom of Saudi Arabia

Dr. Mohamed Fadul A. Elagib BDS, MDS (Perioodntics)

Asst. Professor

Division of Periodontics

Dept. of Preventive Dental Sciences College of Dentistry, King Khalid University Kingdom of Saudi Arabia

Correspondence to:

Prof. Dr. Md. Zahid Hossain BDS, PGD \& PhD (Japan) Professor

Dept. of Periodontology, Oral Pathology \& Oral Medicine

City Dental College, Dhaka e-mail: hoszahid@hotmail.com

\title{
Prevalence of Periodontal Diseases among Patients Attending the Outpatient Department at the College of Dentistry, King Khalid University, Abha, Saudi Arabia
}

\begin{abstract}
:
Aims: A retrospective study was performed involving 2739 patients who were attending the College of Dentistry outpatient clinic department of King Khalid University (KKU), Abha, Kingdom of Saudi Arabia (KSA) during the period of 1st January to 31st December, 2009.

Methods: Random data were collected from the patients record files ofthe College of Dentistry who attended from 1st January to 30th December, 2009.
\end{abstract}

Results: Among 2739 patients mean age $32 \pm 13$ (range 11 82) years, males $86.5 \%$ and females $13.5 \%$, were found sufferers of different types of periodontal diseases i.e., gingivitis and periodontitis. Gingivitis cases were found $1722(63.2 \%)$, in which mild gingivitis was $49.8 \%$, moderate gingivitis $45.8 \%$, severe gingivitis $1.97 \%$, acute gingivitis $0.52 \%$ and puberty gingivitis $1.80 \%$. Total number of patients affected with various forms of periodontitis was 1009 $(36.8 \%)$, in which mild periodontitis was $57.4 \%$, moderate periodontitis $36.6 \%$, severe periodontitis $4.95 \%$ and aggressive periodontitis $0.89 \%$. The prevalence and severity of gingivitis was found increased with age to a peak in the 21 30 years age group, whereas, periodontitis increased significantly $(p<0.001)$ in subjects above the age of 40 years.

Conclusion: Using the research results, a greater effort can be made in providing periodontal health to the population of at or around the city of Abha, KSA.

Key words: Prevalence, gingivitis, periodontitis.

Introduction:

Tooth mortality, which is mainly a reflection of untreated dental caries and periodontal disease, is considered a crude but useful measure for the dental status of a community. Epidemiological studies that have been performed in many parts of the world indicate that periodontal diseases of varying severity are of nearly universal in both children and adolescents. ${ }^{1,2}$ The prevalence of gingivitis is virtually $100 \%$ in a population with no oral hygiene and declines with improved oral hygiene. ${ }^{1-3}$ The prevalence of periodontitis has been elucidated by the World Health Organization (WHO) presenting data on periodontal condition from many countries in its global oral data bank using the community periodontal index of treatment needs (CPITN) criteria. Reports from different places around the world showed a prevalence of severe periodontitis in around $8-10 \%$ of the Population; Sweden 8\%, England 7\%, The Netherlands $10 \%$, Italy $9.6 \%$ and Srilanka $8 \% .^{1-3}$ In India, periodontal diseases are the main cause of dental extractions. ${ }^{1}$

Using data from the 1999-2002 to 2004 National Health and Nutrition Examination Survey (NHANES), United States of America (USA), Researchers demonstrated that adults with low income and less than a high school education are approximately twice as likely to have periodontal diseases compared with more affluent adults with higher educational attainment. ${ }^{5,6}$ 
Physicians should be concerned about the high rates of tooth loss and periodontal diseases because these oral conditions are associated with significant negative health outcomes.4-6Periodontal diseases have potential effects on a wide range of organ systems, e.g., coronary heart disease (CHD), atherosclerosis, myocardial /cerebral infarction, stoke, diabetes mellitus, preterm labor /low birth-weight (LBW) baby, chronic obstructive pulmonary disease, acute respiratory infections and so on. ${ }^{4,5}$

\section{Research Hypothesis:}

A very limited number of epidemiological studies have been carried out to determine the prevalence of oral diseases in the Kingdom of Saudi Arabia. ${ }^{2}$ In a study conducted with 15 85 years of age patients at the dental screening clinics of the College of Dentistry, King Saud University in Riyadh between 1990 and 1992, Investigators found periodontal diseases were the major factors in patients over 40 years of age accounting $51 \%{ }^{2}$ Whereas, in USA, NHANES $1990-$ 2004 report showed only $8.52 \%$ of adults aged $20-64$ years had periodontal diseases and $5.08 \%$ with moderate and severe periodontal diseases. ${ }^{6}$

The prevalence is the number of cases in a designated population at a given point. Any prevalence information must be interpreted in light of the population studied. It was the first effort of the College of Dentistry, KKU, to conduct a research regarding periodontal status among the dental patients thatattended the OPD from 1st January to 30th December, 2009. Two thousand seven hundred and thirty four (2734) dental patients were randomly selected from the College of Dentistry, OPD, KKU. Using the research results, a greater effort can be made in providing periodontal health to the population at or around the city of Abha, KSA.

\section{Subjects and Methods:}

Periodontal disease is defined as having at least one periodontal site with 3 millimeters or more of attachment loss (CAL) and 4 millimeters or more of pocket depth (PPD). using the American Academy of Periodontology (AAP) definition of moderate and severe periodontitis: Moderate periodontal disease is defined as having at least two teeth with interproximal CAL of 4 millimeters or more at least two teeth with 5 millimeters or more of PPD at interproximal sites. Severe periodontal disease is defined as having at least two teeth with interproximal CAL of 6 millimeters or more and at least one tooth with 5 millimeters or more of PPD at interproximal sites. In this study, random data were collected from the patients record files that attended from January 1st to December 30th, 2009 where periodontal diseases were indexed as plaque index $(\mathrm{PI})$, gingival index $(\mathrm{GI})$, calculus index (Cl) and clinical attachment loss (CAL) after (Loe H. J Periodontol1967;38(suppl):610) and modified from (Russel AL. J Dent Res 1956;35:350).

\section{Data analysis:}

Statistical analysis was performed with IBM mainframe version of SAS (statistical analysis system) utilizing the chi-square distribution. The significance value was set as $p<0.05$.

\section{Results:}

Two thousand seven hundred and thirty nine (2739) record files in the OPD, College of Dentistry, were checked for the presence of periodontal diagnosis of which all were sufferers of different forms of periodontal diseases. Average age of the patients was $32 \pm 13$ (range 11-82) years. Males were 2369(86.49\%), mean age $32.1 \pm 50.9$ (range11-82) years and females, 370 $(13.51 \%)$, mean age $34.1 \pm 33.9$ (range $12-80$ ) years (Table -1) indicating a clear predilection $(p<0.001)$ in the male patients. Out of 2739 patients, 1722 (62.94\%), mean age $26 \pm 26.8$ (range 11-82) years, were found having various forms of gingivitis. They were diagnosed as chronic generalized mild gingivitis (49.8\%), moderate gingivitis $(45.8 \%)$, severe gingivitis $(1.92 \%)$, puberty gingivitis $(1.80 \%)$ and acute gingivitis $(0.52 \%)$ (Fig. 1 ). The prevalence and severity of chronic mild and moderate gingivitis found increased with age to a peak in the 21-30 years age group and dramatically declined in the 31-40 years age group, suggesting that gingivitis was prevalent $(p<0.001)$ among the adolescents. Whereas, patients diagnosed with periodontitis were $1009(36.88 \%$ ), mean age $42.8 \pm 7.7$ (range $11-82$ ) years, chronic generalized mild periodontitis $57.4 \%$, moderate periodontitis $36.6 \%$, severe periodontitis $(4.95 \%)$ and aggressive periodontitis $(1.80 \%)$, were found increasing gradually with age and predominantly above the age of 40 years with chronic generalized moderate periodontitis being more common among that age group $(p<0.001)$ (Fig.2).

Out of the 2739 patients, Saudi nationals $1843(71.79 \%)$ were far most the largest population among all nationals including Non-Saudi nationals that was only $896(32.71 \%)$. In non-Saudi nationals, Egyptians 256(9.4\%), Yemenis 229(8.4\%), Pakistanis 90(3.3\%), Bangladeshis $83(3.1 \%)$, Sudanese $58(2.1 \%)$, Indians $54(2.0 \%)$, Filipinos $50(1.8 \%)$, Jordanians $30(1.1 \%)$, Afghanis $10(0.4 \%)$, Turkish $8(0.2 \%)$, Moroccans $7(0.3 \%)$, Nepalese $3(0.1 \%)$, Nigerians $3(0.1 \%)$, Palestinians $3(0.1 \%)$, Srilankanese $3(0.1 \%)$, Indonesians $2(0.1 \%)$, Syrians $2(0.1 \%)$, American $1(0.01 \%)$, Ethiopian $1(0.01 \%)$ and Mauritian $1(0.01 \%)$ (Fig.3). Among the different nationalities, Saudi nationals were found suffering from periodontal diseases more $(p<0.001)$ than any other nationals. The total number of Saudi patients $1843(71.79 \%)$, in which males were $1583(83 \% \pm 50.9)$ and females $257(14 \% \pm 48)$. Saudi nationals were suffering more cases of gingivitis than non-Saudis $(p<0.001)$. The total number of Saudi patients that affected by various forms of gingivitis were $1323(71.90 \pm 16.65 \%)$, whereas, in periodontitis, the total number of affected patients were $520(28.26 \pm 26.28 \%$ ) (Table-2). 
Table-1: Account of total number of patients and periodontal diseases.

\begin{tabular}{|c|c|c|}
\hline Total No. of Patients & $\begin{array}{l}\text { Total No. of } \\
\text { Gingivitis }\end{array}$ & $\begin{array}{l}\text { Total No. of } \\
\text { Periodontitis }\end{array}$ \\
\hline 2739 & 1722 & 1009 \\
\hline M- $2369(86.49 \pm 50.90 \%) \quad F-370(13.51 \pm 33.9 \%)$ & $(62.94 \pm 26.80 \%)$ & $(36.88 \pm 7.70 \%)$ \\
\hline
\end{tabular}

N.B. : M-male, F-female.

Fig.1: Distribution of different types of gingivitis among age groups.

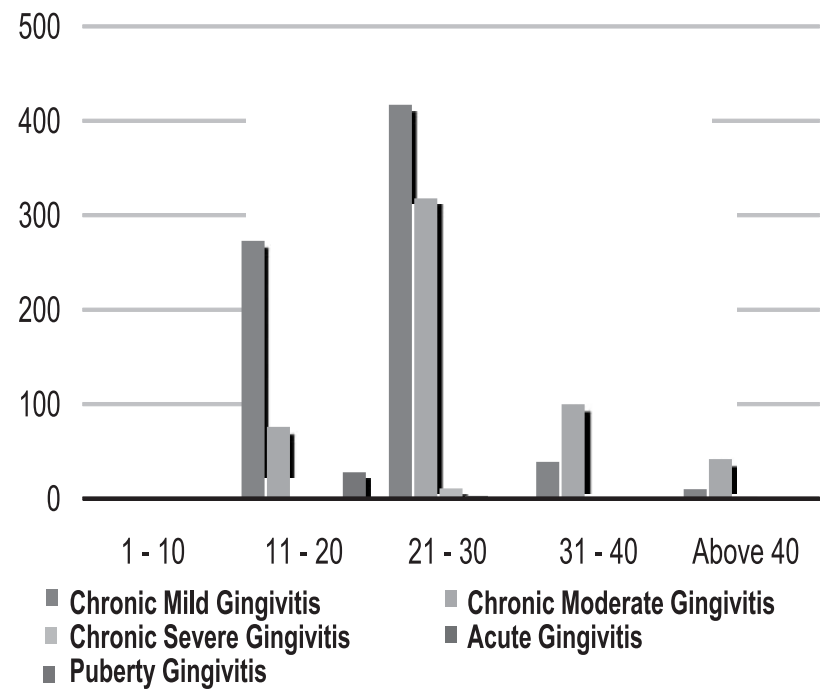

Table-2: Account of all Saudi patients and periodontal diseases among them.

\begin{tabular}{|c|c|c|}
\hline Total No. of Saudi Patients & $\begin{array}{c}\text { Total No. of } \\
\text { Gingivitis }\end{array}$ & $\begin{array}{c}\text { Total No. of } \\
\text { Periodontitis }\end{array}$ \\
\hline 1843 & 1323 & 520 \\
M- $1583(86 \% \pm 50.90) \quad F-257(14 \% \pm 48)$ & $(71.90 \pm 16.65 \%)$ & $(28.26 \pm 26.28 \%)$ \\
\hline
\end{tabular}

N.B. : M -male, F-female.

Fig. 2: Distribution of different types of periodontitis among age groups.

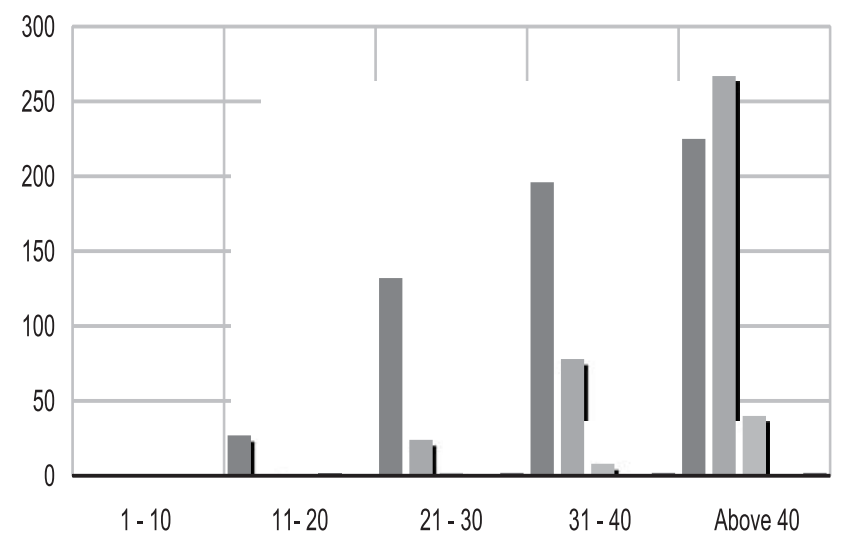

- Chronic Mild Periodontitis \& Chronic Moderate Periodontitis In Chronic Severe Periodontitis I Juvinile Periodontitis - Agreesive Periodontitis
Fig.3: Nationalities of the subjects.

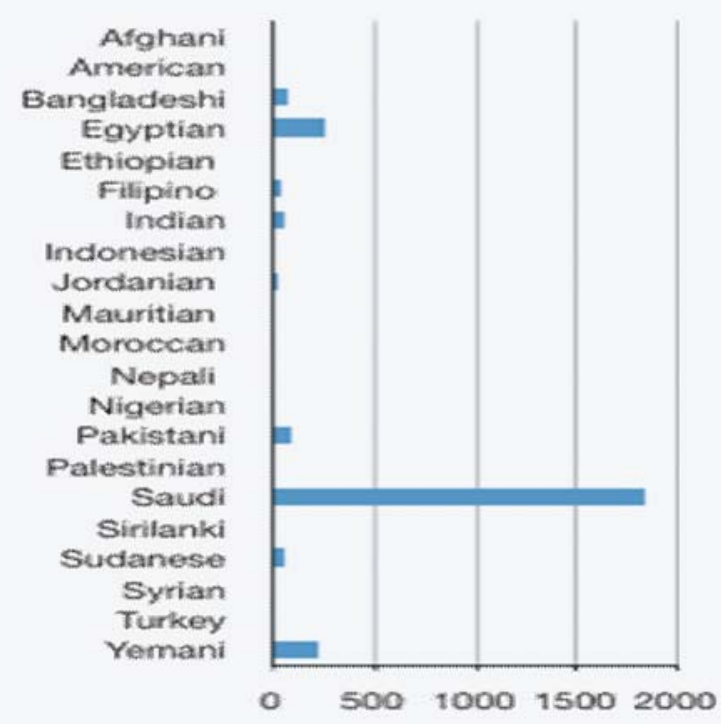

Discussion:

The prevalence is the number of cases in a designated population at a given point. Saudi Arabia is a country of multinational inhabitants and the Abha city of Asir region is not out of this premise. Though various nationalities existed, were not permanent in Abha city. Thus, the main aim of this study was considered for measuring the prevalence of periodontal diseases among Saudi nationals. Among the multi nationalities, Saudi nationals were found $67.3 \%$ that attended the College of Dentistry, KKU in the year of 2009. Of these, male $(86 \%)$ and female $(14 \%)$ patients were found suffering from gingivitis $71.9 \%(p<0.001)$ and periodontitis $29.1 \%$, $(p<0.001)$, respectively (Table-2).

A great number of population were found suffering from gingivitis $(63.2 \%)$ and periodontitis (36.8\%) (Table-1) that is comparable with the published data $(51 \%)$ of the College of Dentistry, King Saud University in Riyadh between 1990 19922 and NHANES, USA 1990 2004 report (8.52\% and 5.08\%)6. Oral hygiene indices (PI, GI, Cl and CAL) were measured after Loe $H(1967)$ (data not shown) that delineated an alarming situation of poor oral hygiene care taken by the patients of at or around Abha city. Female patients were attended a few in number $(14.4 \%)$ that might be a reflection of that the women were not much aware of visiting even the nearby dental hospitals.

\section{Conclusions:}

Using the research results, a greater effort can be made in providing periodontal health to the population of at or around the city of Abha, Kingdom of Saudi Arabia.Systemic diseases and environmental or genetic risk factors were not included in this study. A further broad scale study is needed to measure an accurate prevalence of periodontal diseases among the patients of at or around Abha city. 


\section{Acknowledgment:}

The Authors express their gratitude and acknowledgement to Dr. Ossama A. Mostafa, Associate Professor, Department of Family and Community Medicine, College of Medicine, KKU for his cordial cooperation in analyzing the data.

\section{References:}

1. Bergenholtz A, Jorkjend L. Some modern aspects of periodontal disease. The Saudi Dent J 1990;2(4):156-164.

2. Assery M, Awartani FA. Level of periodontal health knowledge among high school students in the east of Saudi Arabia. The Saudi Dent J 1998;10(3):116-122.

3. Al-Khateeb TL, Al-Amoudi NH, Fatani $\mathrm{HH}$, et.al. Periodontal diseases and caries experience of Diabetic patients in an Arabian community. The Saudi Dent J 1990;2(3):91-95.

4. Committee on Research, Science, and Therapy of the American Academy of Periodontology: position paper: Academy report on epidemiology of periodontal diseases. J Periodontol 2005;76:1406-1419.

5. National health and nutrition examination survey (NHANES); 1999 - 2002.

6. National health and nutrtion examination survey (NHANES); 1999-2004. 